\title{
RESEARCH
}

Open Access

\section{Short- and medium-term outcomes of intracorporeal versus extracorporeal anastomosis in laparoscopic right colectomy: a propensity score-matched study}

Chun-Kai Liao', Yih-Jong Chern', Yueh-Chen Lin', Yu-Jen Hsu', Jy-Ming Chiang ${ }^{1,2}$, Wen-Sy Tsai ${ }^{1,2}$, Pao-Shiu Hsieh ${ }^{1,2}$, Hsin-Yuan Hung ${ }^{1,2}$, Chien-Yuh Yeh ${ }^{1,2}$ and Jeng-Fu You ${ }^{1,2^{*}}$

\begin{abstract}
Backgrounds: Though better short-term outcomes were frequently reported, differences in specimen parameters and the rate of subsequent peritoneal recurrence between intracorporeal anastomosis (IA) and extracorporeal anastomoses (EA) for laparoscopic right hemicolectomy have not been analyzed. We aimed to compare the pathologic differences and oncological outcomes between these two approaches.

Methods: We retrospectively analyzed 217 consecutive patients who underwent laparoscopic right hemicolectomies from September 2016 to April 2018 and classified them into IA and EA groups, based on the approach used. Propensity score matching analysis was performed, after which 101 patients were included in each group with the patients matched for demographics, tumor stage, and localization.
\end{abstract}

Results: The IA group had a longer operative time, shorter length of stay, shorter time to first flatus and tolerating a soft diet, and better pain scale scores at postoperative day 3. No inter-group differences in conversion, postoperative complication, mortality, or readmission rates were found. The IA group had a longer resected colon length (23.67 vs. $19.75 \mathrm{~cm}, p=0.010$ ) and nearest resected margin ( $7.51 \mathrm{vs.} 5.40 \mathrm{~cm}, p=0.010)$ for cancer near the hepatic flexure. There are comparable 3-year overall survival $(87.7 \%$ vs. $89.6 \%, p=0.604)$ and disease-free survival (75.0\% vs. $75.7 \%, p=0.842)$ between the IA and EA groups. The rate of peritoneal recurrence was similar between the two groups ( $5.9 \%$ vs. $7.9 \%, p=0.580)$.

Conclusions: The overall survival, disease-free survival, and the rate of peritoneal recurrence were comparable between the IA and EA procedures. IA ensures better recovery and comparable complications to EA and achieved a more precise tumor excision; thus, IA can be considered a safe procedure for patients with right-sided colon lesions.

Keywords: Right hemicolectomy, Laparoscopic surgery, Propensity score, Resection margin, Intracorporeal anastomosis, Peritoneal recurrence

\footnotetext{
*Correspondence: you3368@adm.cgmh.org.tw

'Colorectal Section, Department of Surgery, Chang Gung Memorial Hospital,

No. 5, Fuxing St., Guishan Dist, Taoyuan 33305, Taiwan

${ }^{2}$ School of Medicine, Chang Gung University, No. 259, Wenhua 1st Road,

Guishan Dist, Taoyuan 33302, Taiwan
}

(c) The Author(s). 2021 Open Access This article is licensed under a Creative Commons Attribution 4.0 International License, which permits use, sharing, adaptation, distribution and reproduction in any medium or format, as long as you give appropriate credit to the original author(s) and the source, provide a link to the Creative Commons licence, and indicate if changes were made. The images or other third party material in this article are included in the article's Creative Commons licence, unless indicated otherwise in a credit line to the material. If material is not included in the article's Creative Commons licence and your intended use is not permitted by statutory regulation or exceeds the permitted use, you will need to obtain permission directly from the copyright holder. To view a copy of this licence, visit http://creativecommons.org/licenses/by/4.0/. The Creative Commons Public Domain Dedication waiver (http://creativecommons.org/publicdomain/zero/1.0/) applies to the data made available in this article, unless otherwise stated in a credit line to the data. 


\section{Introduction}

A laparoscopy-assisted colectomy (LAC) is currently the standard management for benign or malignant colorectal lesions [1-4]. With advancements in surgical instruments and staple devices, total laparoscopic colectomy (TLC) is being performed increasingly for lesions arising from the left side of the colon and rectum. However, for lesions located on the right side, TLC is limited because of the need for intracorporeal hand-sewing of the anastomosis, which is technically more difficult than extracorporeal procedures. Moreover, increasing the surgical time and risk of intra-abdominal contamination with bowel content and tumor cells are major concerns. Many studies have demonstrated the advantages of laparoscopic right hemicolectomy with intracorporeal anastomosis (IA) in terms of short-term outcomes [5-8]. Some advantages of IA are that it helps avoid traction of the bowel through the small laparotomy wound and achieves greater lymph node yields and specimen lengths $[8,9]$. We hypothesize that another advantage of IA is its potential assistance in the precise excision of a tumor, which contributes to a better surgical margin. However, currently, only few studies have analyzed the differences in specimen parameters with most studies presenting a longer specimen length acquired by using IA [8-11], and insufficient data are available regarding the resection length of tumor or the margin length according to different tumor locations, between IA and extracorporeal anastomosis (EA). Additionally, although comparable long-term oncologic outcomes between IA and EA have been reported $[7,12]$, the data are limited in disease-free status and cannot reflect the influence of local recurrence or distant metastasis distinctly.

In this study, the primary outcome was to analyze whether IA achieved a better specimen quality than EA by assessing the specimen parameters. The secondary outcome was to elucidate the postoperative morbidity, recovery, and short- and medium-term oncologic outcomes of laparoscopic right hemicolectomy with IA and compare the results to those of laparoscopic right hemicolectomy with EA.

\section{Materials and methods}

\section{Patient selection}

A total of 217 consecutive patients diagnosed with colon cancer who underwent an elective laparoscopic right hemicolectomy at Chang Gung Memorial Hospital between September 2016 and April 2018 were retrospectively analyzed. Among them, 2 patients without bowel anastomoses, owing to multiple comorbidities, were excluded.

\section{Operative technique}

Pneumoperitoneum was created using the Veress needle technique or mini laparotomy at the umbilicus, which was decided according to the surgeon's preference or the presence of a previous operative scar. Four ports were used including an 11-mm trocar below the umbilicus as a camera port, a 12-mm trocar in the left upper quadrant in the left mid-axillary line as a working port for the stapling device, and two 5-mm trocars in the left and right lower quadrants as working ports. A medial-tolateral approach for mobilization of the right colon and corresponding mesentery was performed in all patients. A standard approach following the concept of complete mesocolic excision and central vascular ligation was performed. The ileocolic vessel was first ligated with the Hem-o-lok ${ }^{\diamond}$ System (Weck Closure Systems, Research Triangle Park, NC, USA) at its root from Superior mesentery vessels. Then, a medial-to-lateral, bottom-to-up dissection of retroperitoneum to lateral attachment of ascending colon and hepatic flexure was performed. After completely dividing the mesentery of the planned resected bowel, including the marginal artery, the ileocolic anastomosis was performed by either EA or IA according to the surgeon's preference.

In the EA group, the umbilical wound was extended to a length of $5-7 \mathrm{~cm}$, and a wound protector was used. The right side of the colon and terminal ileum were exteriorized through the midline incision, and the ileocolic anastomosis was created either using the antiperistaltic side-to-side stapler method or the isoperistaltic end-toend hand-sewn method.

In the IA group, both ends of the transverse colon and terminal ileum were divided with Endo GIA staplers (Medtronic, Minneapolis, USA) laparoscopically. One of the following anastomosis methods was chosen according to the surgeon's preference: (a) an isoperistaltic endto-side hand-sewn method, (b) an isoperistaltic side-toside staple method, and (c) an antiperistaltic side-to-side stapler method. For the isoperistaltic staple method, one enterotomy was created on the antimesenteric side of the ileum and colon, followed by insertion of the jaws of the staples and creation of the anastomosis. The common channel of the enterotomy was then closed with a barbed knotless suture by 3-0 V-Loc (Medtronic, Minneapolis, USA). For the antiperistaltic side-to-side stapler method, the enterotomy was created near the transection side, followed by a side-to-side staple anastomosis; the common channel of the enterotomy was also closed by stapling. After anastomosis, the specimen was extracted mainly through the Pfannenstiel incision or by extending the right lower quadrant port wound. For patients with a previous midline surgery, the umbilical wound was extended from the previous scar.

Natural orifice specimen extraction (NOSE) was also an alternative method. For female patients, a transvaginal or transrectal NOSE procedure was chosen according to the surgeon's preference. For transvaginal 
approach, the posterior vagina was opened and a double-ringed wound protector (Alexis wound retractor; Applied Medical, Rancho Santa Margarita, CA, USA) was used to protect and shorten the vaginal canal after cleaning the vagina with povidone-iodine solution. After pulling out the specimen through the vaginal canal, the colpotomy incision was closed with an absorbable suture. For transrectal approach, rectosigmoid colon lumen was blocked using a bowel clamp followed by rectal irrigation with povidone-iodine solution. One enterotomy was made at the upper rectum, and a doubleringed wound protector was inserted to protect and shorten the rectum. After retrieval of specimen, the rectal opening was closed by barbed suture, and the air leak test was performed to confirm no mechanical failure. For male patients, transrectal NOSE can be used.

\section{Data collection}

A retrospective analysis of data from the Colorectal Section Tumor Registry in Chang Gung Memorial Hospital, a prospectively designed database consisting of the records of postoperative patients who were consecutively and actively followed up, was conducted. Written informed consent was obtained from the patients prior to study participation. This study was approved by the Institutional Review Board of Chang Gung Memorial Hospital (approval number 202000106B0) and performed in accordance with the Declaration of Helsinki. All data were recorded in the hospital database and used for research purposes.

Preoperative variables, including age, sex, body mass index, comorbidity, history of previous abdominal surgery, American Society of Anesthesiologists (ASA) Class, and albumin level, were analyzed. The blood sample was obtained 1 week prior to the surgery. The operative parameters including the operative method, operative time, concomitant surgery, conversion to open surgery, blood loss, anastomosis methods, and specimen retraction site were collected. The pathological parameters including tumor location, tumor size, number of harvested lymph nodes, length of resection margins, $\mathrm{T}$ stage, and $\mathrm{N}$ stage were also analyzed. A subgroup analysis of the surgical margin according to the tumor location (cecum, ascending colon, hepatic flexure, and transverse colon) was also performed. The outcomes retrieved to compare the IA and EA groups included complications, mortality, length of hospital stay, readmission within 30 days of discharge, and pain scale scores at postoperative days 1 to 3 . Time to first flatus or stool passage and time to tolerating a liquid and soft diet were also analyzed.

\section{Follow-up}

All physicians at the author's institution adopted similar follow-up routines and adjuvant treatment protocols.
After primary tumor resection, all patients were subjected to a follow-up program that included outpatient visits every 3 to 6 months for physical examinations and CEA tests. Chest radiography, abdominal ultrasonography, or abdominal computed tomography (CT) imaging, in addition to colonoscopy, were performed after surgery and every 1 to 3 years whenever necessary. Follow-up status was confirmed postoperatively every 12 months by a team of three physicians and five specially trained nurses. Date of first recurrence was defined as the first date when the existence of local recurrence and/or distant metastases was confirmed by histology of biopsy specimens, additional surgery, and/or by radiological studies. The index date for survival calculation was the date of surgery for colon cancer. The definition of locoregional recurrence in our study is recurrent lesions within or adjacent to the original tumor location or dissection plane. Distant metastasis is defined as metastasis to non-locoregional sites like the liver, lung, or other organs. The presence of peritoneal recurrence was confirmed by either CT/positron emission tomography (PET) findings and/or surgical findings. The last followup date in this study was July 31, 2020.

\section{Statistical analysis}

All parameters were analyzed using the Statistical Package for Social Sciences (SPSS) version 24 (IBM Corp., Armonk, New York, USA). Propensity score matching (PSM) was performed using a logistic regression model with the anastomosis method (IA vs. EA) set as the dependent variable. Patients were matched $1: 1$ by neighbor matching methods. The categorical variables were compared using Pearson's chi-squared test, whereas continuous variables were compared using the independent sample $t$ test. Survival analysis was performed using Kaplan-Meier curves with the log-rank test. $P<0.05$ was considered statistically significant.

\section{Results}

Patient characteristics before and after matching

The total number of study patients and the number of patients in each study group before and after propensity score adjustment is presented in Fig. 1. Of the 215 patients included in the analysis, 114 and 101 patients underwent extracorporeal ileocolic anastomosis (EA group) and intracorporeal ileocolic anastomosis (IA group), respectively. The demographics of these patients are shown in Table 1. No significant differences were found between the EA and IA groups, except the preoperative albumin levels. There were more patients with low preoperative albumin levels in the EA group than in the IA group $(<3.5 \mathrm{~g} /$ $\mathrm{dL}, 13.3 \%$ vs. $3 \%, p=0.007)$. After PSM, the study population included 101 patients in each group 


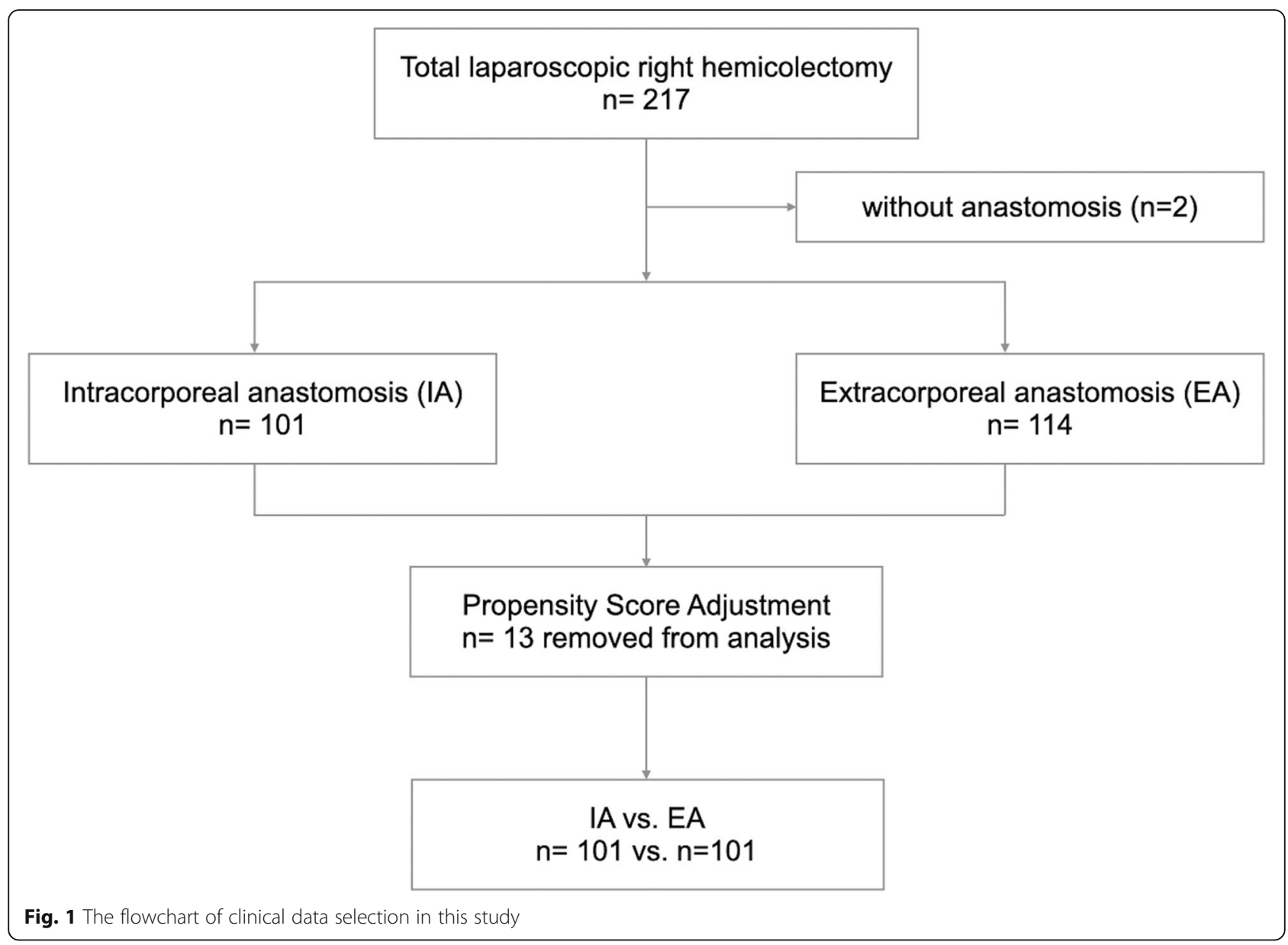

(Table 1). Baseline characteristics were not significantly different between the two groups.

\section{Operative parameter}

The operative parameters are listed in Table 2. There were no significant differences in the operative method, concomitant surgery, and blood loss between the two groups. Both groups had no case of conversion to laparotomy. The mean operative time was significantly shorter in the EA group than in the IA group (195.5 vs. $219.0 \mathrm{~min}, p=0.034)$. There were various methods used to perform the ileocolic anastomosis in both groups, but the IA group predominantly used the method utilizing a staple with a barbed suture (56.4\%). The incision made for specimen extraction was significantly different between the groups. In the EA group, all specimens were retracted from the trans-umbilical wound. In the IA group, a Pfannenstiel incision was made in $68.3 \%$ of the patients. The other incisions made included a transumbilical wound (8.9\%), McBurney's incision (18.8\%), and NOSE (4\%).

\section{Pathological parameters}

There were no significant differences in the tumor size, tumor location, number of harvested lymph nodes, stage of tumor invasion, and stage of lymph node invasion between the EA and IA groups (Table 3). The colon lengths were significantly different between the EA and IA groups $(17.45 \pm 5.81$ and $20.36 \pm 6.78 \mathrm{~cm}$, respectively; $p=0.010)$; however, differences in the ileum length and nearest margin length were not statistically significant (Table 4).

In the subgroup analysis according to tumor location, a trend of increasing colon length but decreasing ileum length was observed in patients with tumors located from the cecum to the transverse colon in both groups (Fig. 2a). For tumors located from the hepatic flexure to the transverse colon, a significant difference in colon length was found between the EA and IA groups (19.75 \pm 6.67 and $23.67 \pm 5.95 \mathrm{~cm}$, respectively; $p=0.010$ ). There was also a significant difference in the length of the nearest margin between the EA and IA groups (5.40 \pm 2.83 and $7.51 \pm 3.81 \mathrm{~cm}$, respectively; $p=0.010$ ) (Fig. 2b). Although no significant difference was observed in 
Table 1 Demographic characteristics of patients undergoing intracorporeal and extracorporeal anastomosis before and after propensity score matching

\begin{tabular}{|c|c|c|c|c|c|c|}
\hline & \multicolumn{3}{|c|}{ Before propensity score matching } & \multicolumn{3}{|c|}{ After propensity score matching } \\
\hline & $\mathrm{IA}^{\mathrm{a}}(n=101)$ & $\mathrm{EA}^{\mathrm{b}}(n=114)$ & $P$ value & $\mathrm{IA}^{\mathrm{a}}(n=101)$ & $\mathrm{EA}^{\mathrm{b}}(n=101)$ & $P$ value \\
\hline Age (years) & $64.43 \pm 11.65$ & $67.46 \pm 13.84$ & 0.086 & $64.43 \pm 11.65$ & $66.88 \pm 12.79$ & 0.155 \\
\hline \multicolumn{7}{|l|}{ Sex, $n(\%)$} \\
\hline Male & $57(56.4)$ & $54(47.4)$ & 0.184 & $57(56.4)$ & $46(45.5)$ & 0.122 \\
\hline Female & $44(43.6)$ & $60(52.6)$ & & $44(43.6)$ & $55(54.5)$ & \\
\hline \multicolumn{7}{|l|}{$\mathrm{BMI}^{\mathrm{C}}\left(\mathrm{kg} / \mathrm{m}^{2}\right)$} \\
\hline Mean & $24.24 \pm 3.66$ & $24.14 \pm 4.17$ & 0.852 & $24.24 \pm 3.66$ & $24.45 \pm 4.25$ & 0.715 \\
\hline $\mathrm{BMI}^{\mathrm{C}}>30$ & $6(5.9)$ & $8(7.0)$ & 0.749 & $6(5.9)$ & $8(7.9)$ & 0.580 \\
\hline \multicolumn{7}{|l|}{ Comorbidity, n (\%) } \\
\hline Hypertension & $49(48.5)$ & $54(47.4)$ & 0.867 & $49(48.5)$ & $48(47.5)$ & 0.888 \\
\hline Cardiac disease & $5(5)$ & $12(10.6)$ & 0.138 & $5(5)$ & $11(11.0)$ & 0.113 \\
\hline$C_{V A}{ }^{d}$ & $1(1)$ & $2(1.8)$ & 1.000 & $1(1)$ & $2(2.0)$ & 0.621 \\
\hline Asthma & $1(1)$ & $2(1.8)$ & 1.000 & $1(1)$ & $2(2.0)$ & 0.621 \\
\hline Diabetes & $24(23.8)$ & $24(21.2)$ & 0.659 & $24(23.8)$ & $20(20.0)$ & 0.519 \\
\hline Cirrhosis & $2(2)$ & $0(0)$ & 0.222 & $2(2)$ & $0(0)$ & 0.498 \\
\hline \multicolumn{7}{|l|}{$\mathrm{ASA}^{\mathrm{e}}$} \\
\hline$\|$ & $33(32.7)$ & $36(31.6)$ & 0.864 & $33(32.7)$ & $35(34.7)$ & 0.766 \\
\hline III & $69(67.3)$ & $78(68.4)$ & & $68(67.3)$ & $66(65.3)$ & \\
\hline Previous abdominal operation & 19 (18.8) & $30(26.3)$ & 0.191 & $19(18.8)$ & $24(23.8)$ & 0.390 \\
\hline \multicolumn{7}{|l|}{ Albumin } \\
\hline$<3.5 \mathrm{~g} / \mathrm{dl}$ & $3(3)$ & $15(13.3)$ & 0.007 & $3(3)$ & $3(3)$ & 1 \\
\hline$\geq 3.5 \mathrm{~g} / \mathrm{dl}$ & $98(97)$ & $98(86.7)$ & & $98(97)$ & $98(97)$ & \\
\hline
\end{tabular}

Intracorporeal anastomosis

${ }^{\mathrm{b}}$ Extracorporeal anastomosis

'Body mass index

${ }^{\mathrm{d}}$ Cerebrovascular accident

${ }^{\mathrm{e}}$ American Society of Anesthesiologist

the ileum length, we noted that the IA group had a shorter ileum distance from the transection line than the EA group ( $4.49 \pm 2.41$ vs. $6.10 \pm 4.88 \mathrm{~cm} ; p=0.075)$.

\section{Short-term postoperative outcomes}

The postoperative complications and short-term outcomes are listed in Table 5. The overall complication rates were similar between the IA and EA groups (8.9\% vs. $10.9 \%, p=0.818)$. No difference among severe complications (Clavien-Dindo Grade $\geq 3$ ) was observed between the two groups ( $2 \%$ vs. $1 \%, p=0.586)$. The major complication in both groups was prolonged postoperative ileus, with no significant difference observed between the groups. All patients recovered after conservative treatment. Three patients experienced intraabdominal infection and received subsequent parenteral antibiotics treatment. Among them, one patient in the IA group presented with a pelvic abscess, which was confirmed by CT study and was readmitted within 1 month for percutaneous drainage. One patient in the IA group presented with dehiscence of anastomosis and required re-operation. Another patient in the EA group presented with dehiscence of anastomosis after discharge and required readmission for surgical management. One patient in the EA group had upper gastrointestinal bleeding and was readmitted for conservative treatment. One patient with a history of anxiety disorder visited emergency room for dyspnea and was admitted for conservative treatment. No mortality occurred in any of the groups.

Patients who underwent IA had significantly lower pain scale scores at postoperative day 3 than those who underwent EA (2.4 vs. 2.9, $p<0.001$ ). Better recovery was observed in the IA group than in the EA group in terms of time to first flatus ( 2.2 vs. 2.6 days, $p=0.027)$, time to first bowel movement (3.5 vs. 4.4 days, $p<$ 0.001 ), time to tolerating a liquid diet (3.9 vs. 4.9 days, $p$ $=0.017)$, and time to tolerating a soft diet (5.7 vs. 6.7 days, $p=0.039$ ). The mean length of postoperative stay was significantly shorter in the IA group than in the EA 
Table 2 Post-matching of operative parameters of patients undergoing intracorporeal and extracorporeal anastomosis

\begin{tabular}{|c|c|c|c|}
\hline & $I A^{a}(n=101)$ & $\mathrm{EA}^{\mathrm{b}}(n=101)$ & $P$ value \\
\hline \multicolumn{4}{|l|}{ Operation } \\
\hline $\mathrm{RH}^{\mathrm{c}}$ & $96(95)$ & $91(90.1)$ & 0.180 \\
\hline Extended $\mathrm{RH}^{\mathrm{c}}$ & $5(5)$ & $10(9.9)$ & \\
\hline Operative time, min & $219.0 \pm 77.1$ & $195.5 \pm 80.1$ & 0.034 \\
\hline Concomitant surgery & $7(6.9)$ & $13(12.9)$ & 0.158 \\
\hline Conversion to open & $0(0)$ & $0(0)$ & \\
\hline Blood loss > $100 \mathrm{ml}$ & $6(5.9)$ & $10(9.9)$ & 0.297 \\
\hline \multicolumn{4}{|l|}{ Anastomosis methods } \\
\hline Hand sewn & $23(22.8)$ & $34(33.7)$ & $<0.001$ \\
\hline Total stapled & $21(20.8)$ & $67(66.3)$ & \\
\hline Stapled and barbed suture & $57(56.4)$ & & \\
\hline \multicolumn{4}{|l|}{ Extraction site } \\
\hline Trans-umbilical & $9(8.9)$ & $101(100)$ & $<0.001$ \\
\hline Pfannenstiel & $69(68.3)$ & & \\
\hline $\operatorname{NOSE}^{d}$ & $4(4)$ & & \\
\hline McBurney & 19 (18.8) & & \\
\hline
\end{tabular}

${ }^{\text {antracorporeal anastomosis }}$

${ }^{b}$ Extracorporeal anastomosis

${ }^{\mathrm{c}}$ Right hemicolectomy

${ }^{\mathrm{d}}$ Natural orifice specimen extraction

group (7.1 vs. 8.7 days, $p=0.005)$. The proportion of patients in the IA group who had a length of stay (LOS) shorter than 5 days was $39.6 \%$, whereas that in the EA group was $6.9 \%(p<0.001)$.

The follow-up laboratory data on postoperative day 3 revealed that the IA group had more patients with leukocytosis than the EA group (white blood cell [WBC] $\geq 10000 / \mathrm{mm}^{3}: 49 \%$ vs. $\left.28.6 \%, p=0.004\right)$ and a higher Creactive protein (CRP) level (88.1 vs. $63.3 \mathrm{mg} / \mathrm{L}, p<$ 0.001).

\section{Long-term postoperative outcomes}

The long-term outcomes are listed in Table 6. The median follow-up times were 31.93 months and 36.7 months in the IA group and EA group, respectively. Four incisional hernias occurred during follow-up periods in the EA group, whereas no incisional hernia occurred in the IA group. The 3-year overall survival was not significantly different between the IA and EA groups (87.7\% and $89.6 \%$, respectively, $p=0.604$ ). The 3 -year disease-free survival was also not significantly different between the IA and EA groups $(75.0 \%$ and $75.7 \%$, respectively, $p=0.842$ ). In subgroup analysis, there were 6 peritoneal recurrences found in the IA group and 8 in the EA group. The disease-free survival regarding peritoneal recurrence was not different at the 3-year follow-
Table 3 Post-matching of pathologic parameters of patients undergoing intracorporeal and extracorporeal anastomosis

\begin{tabular}{|c|c|c|c|}
\hline & $\mathrm{IA}^{\mathrm{a}}(n=101)$ & $\mathrm{EA}^{\mathrm{b}}(n=101)$ & $P$ value \\
\hline Harvested lymph nodes & $40.9 \pm 19.3$ & $41.1 \pm 19.9$ & 0.940 \\
\hline \multicolumn{4}{|l|}{ Tumor size (cm) } \\
\hline Width & $5.0 \pm 9.7$ & $4.3 \pm 2.2$ & 0.468 \\
\hline Length & $4.5 \pm 9.7$ & $3.3 \pm 1.5$ & 0.242 \\
\hline \multicolumn{4}{|l|}{ Tumor location } \\
\hline Cecum and appendix & 19 (18.8) & $16(15.8)$ & 0.369 \\
\hline Ascending colon & $42(41.6)$ & $52(51.5)$ & \\
\hline $\begin{array}{l}\text { Transverse colon and } \\
\text { hepatic flexure }\end{array}$ & 40 (39.6) & $33(32.7)$ & \\
\hline \multicolumn{4}{|l|}{ T stage } \\
\hline T0 & $5(5)$ & $2(2)$ & 0.778 \\
\hline T1 & $17(16.8)$ & $14(13.9)$ & \\
\hline $\mathrm{T} 2$ & $9(8.9)$ & $11(10.9)$ & \\
\hline T3 & $48(47.5)$ & $55(54.5)$ & \\
\hline T4a & $17(16.8)$ & $15(14.9)$ & \\
\hline $\mathrm{T} 4 \mathrm{~b}$ & $5(5)$ & $4(4)$ & \\
\hline \multicolumn{4}{|l|}{ N stage } \\
\hline No & $57(56.4)$ & $72(71.3)$ & 0.286 \\
\hline N1a & $15(14.9)$ & $9(8.9)$ & \\
\hline $\mathrm{N} 1 \mathrm{~b}$ & $9(8.9)$ & $8(7.9)$ & \\
\hline N1c & $2(2)$ & $1(1)$ & \\
\hline $\mathrm{N} 2 \mathrm{a}$ & $5(5)$ & $5(5)$ & \\
\hline $\mathrm{N} 2 \mathrm{~b}$ & $13(12.9)$ & $6(5.9)$ & \\
\hline
\end{tabular}

Intracorporeal anastomosis

${ }^{b}$ Extracorporeal anastomosis

up between the IA and EA groups (81.6\% and $82.9 \%$, respectively, $p=0.923$ ) (Fig. 3a and $\mathrm{b}$ ).

\section{Discussion}

Nowadays, with innovations in surgical instrumentation, IA using linear staples and barbed suture material allows for the increasing application of total laparoscopic right hemicolectomies. In 2008, Bergamaschi et al. described one standardized IA procedure performed during a right colectomy for cancer, which showed favorable shortterm results [5]. However, many surgeons still hesitate to use IA for laparoscopic right hemicolectomy, and EA remains the method of choice. Besides its technical difficulty, some concerns exist, including the possibility of increasing the risk of infection and exposure of tumor cell with this technique because the intestinal tract is opened during the anastomosis.

In this study, we compared the outcomes of patients who underwent laparoscopic right hemicolectomy for right-sided colon cancer using IA with that of those who underwent laparoscopic right hemicolectomy for right- 
Table 4 Post-matching of the specimen length of patients undergoing intracorporeal and extracorporeal anastomosis according to tumor location

\begin{tabular}{|c|c|c|c|c|}
\hline Tumor location & Specimen length $(\mathrm{cm})$ & $\mathrm{IA}^{\mathrm{a}}(n=101)$ & $\mathrm{EA}^{\mathrm{b}}(n=101)$ & $P$ value \\
\hline \multirow[t]{4}{*}{ All } & Colon, mean & $20.36 \pm 6.78$ & $17.45 \pm 5.81$ & 0.010 \\
\hline & Ileum, mean & $5.70 \pm 3.33$ & $7.26 \pm 7.90$ & 0.760 \\
\hline & Nearest margin, mean & $7.35 \pm 3.67$ & $6.62 \pm 3.10$ & 0.130 \\
\hline & & $\mathrm{IA}^{\mathrm{a}}(n=19)$ & $\mathrm{EA}^{\mathrm{b}}(n=16)$ & \\
\hline \multirow[t]{4}{*}{ Cecum and appendix } & Colon, mean & $15.92 \pm 4.63$ & $14.33 \pm 4.49$ & 0.311 \\
\hline & Ileum, mean & $8.70 \pm 4.76$ & $7.93 \pm 6.11$ & 0.686 \\
\hline & Nearest margin, mean & $7.66 \pm 4.87$ & $6.82 \pm 3.11$ & 0.569 \\
\hline & & $1 A^{a}(n=42)$ & $\mathrm{EA}^{\mathrm{b}}(n=52)$ & \\
\hline \multirow[t]{4}{*}{ Ascending colon } & Colon, mean & $19.22 \pm 6.92$ & $16.96 \pm 5.06$ & 0.071 \\
\hline & lleum, mean & $5.61 \pm 2.61$ & $7.75 \pm 9.73$ & 0.175 \\
\hline & Nearest margin, mean & $7.07 \pm 2.98$ & $7.33 \pm 3.08$ & 0.678 \\
\hline & & $\mathrm{A}^{\mathrm{a}}(n=40)$ & $\mathrm{EA}^{\mathrm{b}}(n=33)$ & \\
\hline \multirow[t]{3}{*}{ Transverse colon and hepatic flexure } & Colon, mean & $23.67 \pm 5.95$ & $19.75 \pm 6.67$ & 0.010 \\
\hline & lleum, mean & $4.49 \pm 2.41$ & $6.10 \pm 4.88$ & 0.075 \\
\hline & Nearest margin, mean & $7.51 \pm 3.81$ & $5.40 \pm 2.83$ & 0.010 \\
\hline
\end{tabular}

Intracorporeal anastomosis

${ }^{b}$ Extracorporeal anastomosis

sided colon cancer using EA. Surgical time was found to be significantly longer in the IA group than in the EA group. This is mainly attributed to the need for handsewing and knotting inside the abdominal cavity, and many surgeons required further training to perform intracorporeal sutures efficiently. The longer surgical time required to perform IA is also mentioned by several studies $[5,8,13,14]$; however, we predict that this may shorten with increased surgical experience and the establishment of a standard approach. Given that EA requires grasping of the colon and ileum for subsequent anastomosis, a trans-umbilical incision is usually chosen and the length of the wound is inevitably longer; however, using IA, any incision site can be selected for specimen retraction and the length of the wound can be shorter for grasping one longer specimen. Many studies have revealed that patients with a lower abdominal incision experience less postoperative pain, a quicker return
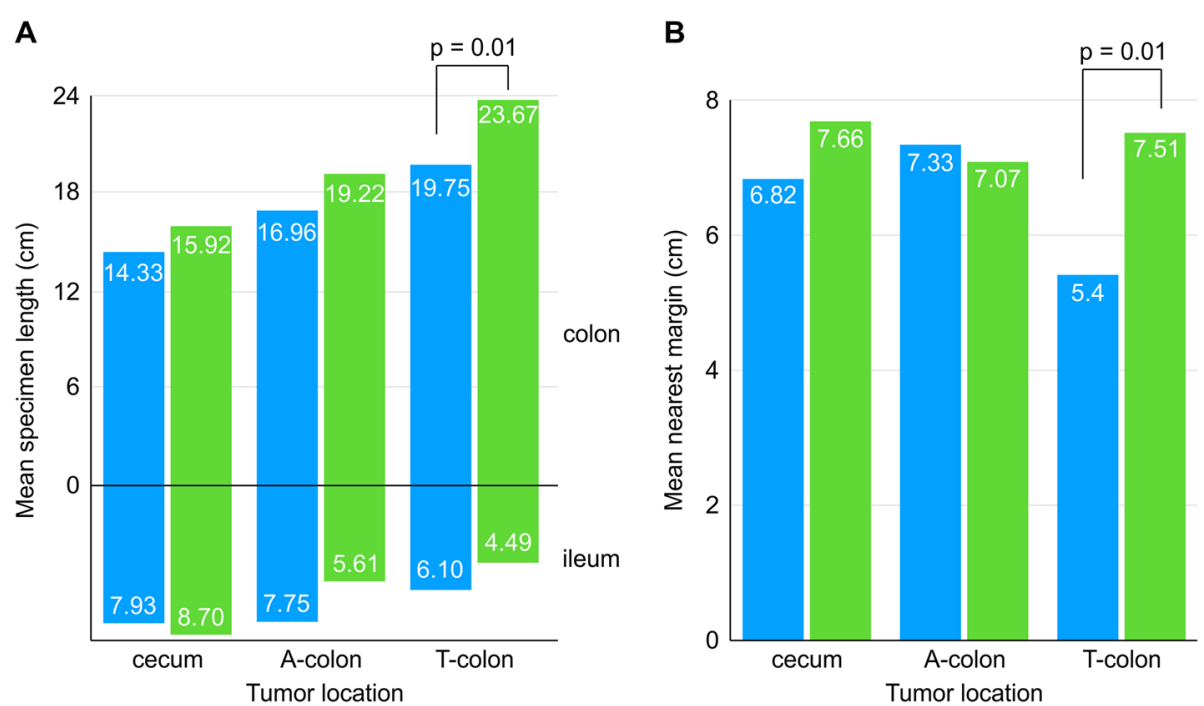

Fig. 2 A comparison of the specimen length between extracorporeal anastomosis and intracorporeal anastomosis according to the tumor location. a Mean specimen length according to tumor location in patients that had extracorporeal anastomosis (EA group; blue bars) versus those that had intracorporeal anastomosis (IA group; green bars). b Mean nearest margin length according to tumor location, in the extracorporeal anastomosis group (EA group; blue bars) versus the intracorporeal anastomosis group (IA group; green bars) 
Table 5 Post-matching of postoperative outcomes of patients undergoing intracorporeal and extracorporeal anastomosis

\begin{tabular}{|c|c|c|c|}
\hline & $\mathrm{IA}^{\mathrm{a}}(n=101)$ & $\mathrm{EA}^{\mathrm{b}}(n=101)$ & $P$ value \\
\hline Total complications & $10(9.9)$ & $11(10.9)$ & 0.818 \\
\hline \multicolumn{4}{|l|}{ Clavien-Dindo classification } \\
\hline Grade 1 & 7 & 7 & \multirow[t]{6}{*}{0.781} \\
\hline Grade 2 & 1 & 3 & \\
\hline Grade 3a & 1 & 0 & \\
\hline Grade 3b & 1 & 1 & \\
\hline Grade 4 & 0 & 0 & \\
\hline Grade 5 & 0 & 0 & \\
\hline \multicolumn{4}{|l|}{ Complication type } \\
\hline Postoperative ileus & $7(6.9)$ & $6(5.9)$ & \multirow[t]{6}{*}{0.857} \\
\hline$|A|^{c}$ & $1(1)$ & $2(2)$ & \\
\hline Dehiscence of anastomosis & $1(1)$ & $1(1)$ & \\
\hline Wound infection & $1(1)$ & $0(0)$ & \\
\hline Others & $0(0)$ & $2(2)$ & \\
\hline Mortality & $0(0)$ & $0(0)$ & \\
\hline \multicolumn{4}{|l|}{ Length of stay } \\
\hline Mean & $7.1 \pm 3.9$ & $8.7 \pm 4.2$ & 0.005 \\
\hline $\operatorname{LOS}^{d} \leq 5$ days & $40(39.6)$ & $7(6.9)$ & $<0.001$ \\
\hline Readmission $<30$ days & $1(1)$ & $2(2)$ & 0.561 \\
\hline \multicolumn{4}{|l|}{ POD-3 data } \\
\hline $\mathrm{WBC}^{\mathrm{e}} \geq 10000 / \mathrm{mm}^{3}$ & $47(49)$ & 28 (28.6) & 0.004 \\
\hline $\mathrm{CRP}^{\mathrm{f}}(\mathrm{mg} / \mathrm{L})$ & $88.1 \pm 49.8$ & $63.3 \pm 41.7$ & $<0.001$ \\
\hline \multicolumn{4}{|l|}{ Pain scale (visual analog scale) } \\
\hline $\mathrm{POD}^{9} 1$ & $4.2 \pm 1.7$ & $4.4 \pm 1.9$ & 0.430 \\
\hline $\mathrm{POD}^{9} 2$ & $3.1 \pm 1.3$ & $3.4 \pm 1.7$ & 0.148 \\
\hline $\mathrm{POD}^{9} 3$ & $2.4 \pm 0.7$ & $2.9 \pm 1.4$ & $<0.001$ \\
\hline Time to first flatus, days & $2.2 \pm 1.2$ & $2.6 \pm 1.1$ & 0.027 \\
\hline Time to first bowel movement, days & $3.5 \pm 1.5$ & $4.4 \pm 2.0$ & $<0.001$ \\
\hline Time to tolerate liquid diet, days & $3.9 \pm 2.6$ & $4.9 \pm 3.6$ & 0.017 \\
\hline Time to tolerate soft diet, days & $5.7 \pm 3.2$ & $6.7 \pm 3.8$ & 0.039 \\
\hline
\end{tabular}

antracorporeal anastomosis

${ }^{b}$ Extracorporeal anastomosis

Intra-abdominal infection

dLength of stay

eWhite blood cell

${ }^{\mathrm{f}} \mathrm{C}$-Reactive protein

gPostoperative day

to ambulation and normal bowel function, and fewer pulmonary complications [15-18]. In our institution, we mainly choose the Pfannenstiel incision for tumor retraction because of its better postoperative outcomes and cosmetic results. In this study, the pain scale (visual analog scale) score showed a significant improvement on postoperative day 3 in the IA group. Moreover, some studies have shown a lower rate of incisional hernias with the use of the Pfannenstiel incision compared to that with an umbilical incision $[16,19,20]$. In our series, no incision hernia was found in the IA group at the 3- year follow-up, which is consistent with the findings of studies from western countries.

There are many advantages of IA for bowel management; one is the better visualization of both limbs of bowel. The EA method requires the bowel to be pulled through a small incision, which may cause small lacerations of the mesentery or torsion of the bowel while manipulating it during externalization. On the other hand, the IA method requires less mobilization of the bowel and can achieve better incisions that allow for a proper surgical margin to be obtained with better visualization 
Table 6 Post-matching of long-term outcomes of patients undergoing intracorporeal and extracorporeal anastomosis

\begin{tabular}{llll}
\hline & $\mathbf{I A}^{\mathbf{a}}(\boldsymbol{n}=\mathbf{1 0 1})$ & $\mathbf{E A}^{\mathbf{b}}(\boldsymbol{n}=\mathbf{1 0 1})$ & $\boldsymbol{P}$ value \\
\hline Follow-up time, median months & $31.93(1.54-44.35)$ & $36.70(2.43-46.23)$ & 0.001 \\
Incisional hernia & $0(0)$ & $4(3.9)$ & $0.121^{\mathbf{C}}$ \\
Peritoneal recurrence & $6(5.9)$ & $8(7.9)$ & 0.580 \\
$\quad$ Primary T3 lesion & $2(33.3 \%)$ & $2(25 \%)$ & 1.000 \\
$\quad$ Primary T4 lesion & $4(66.7 \%)$ & $6(75 \%)$ & $89.6 \%$ \\
3-year overall survival & $87.7 \%$ & $75.7 \%$ & 0.604 \\
3-year disease-free survival & $75.0 \%$ & $82.9 \%$ & 0.842 \\
3-year peritoneal recurrence-free survival & $81.6 \%$ & & 0.923 \\
\hline
\end{tabular}

Intracorporeal anastomosis

${ }^{b}$ Extracorporeal anastomosis

'Fisher's exact test

using a direct-camera view. Less bowel manipulation is thought to enhance bowel function recovery. In this study, the times to first flatus, tolerating a soft diet, and bowel movement were significantly shorter in the IA group, which is similar to that found in other studies $[8$,
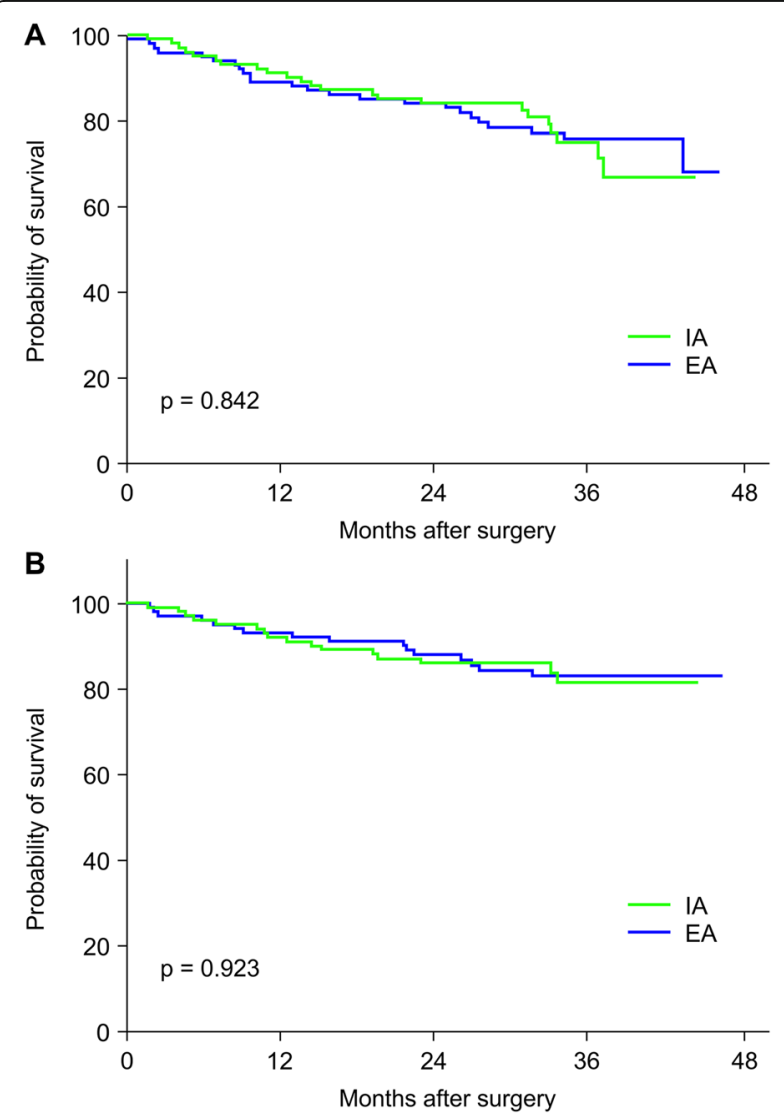

Fig. 3 a Comparison of 3-year disease-free survival between patients who had extracorporeal anastomosis (EA group; blue bars) and those who had intracorporeal anastomosis (IA group; green bars). $\mathbf{b}$ Comparison of 3-year local recurrence-free survival between patients who had extracorporeal anastomosis (EA group; blue bars) and those who had intracorporeal anastomosis (IA group; green bars)
$12,21]$. Another advantage of IA is that it allows for better alignment of the bowel during anastomosis with isoperistaltic ileocolic anastomosis and less inversion of the ileum and its mesentery may also contribute to better recovery of bowel function.

During the pathological review, the number of harvested lymph nodes was not significantly different between the IA and EA groups in our study, although some studies demonstrated a higher number of retrieved lymph nodes with the IA method $[8,12]$. Only a few studies have compared the difference in specimen length and surgical margin between the IA and EA groups. Magistro et al. compared these parameters in 40 patients undergoing a TLC with that of 40 patients undergoing a LAC and found a longer specimen length in the IA group $(37.7 \pm 12 \mathrm{~cm}$ vs. $29.8 \pm 8.2 \mathrm{~cm}, p=0.001)$ [8]. Another study by Biondi et al. revealed that better specimen and vascular pedicle lengths are achieved using the IA method. In this study, a greater colon length was achieved with the IA method than with the EA method (20.36 vs. $17.45 \mathrm{~cm}, p=0.01$ ) [9]. In a single-blind, randomized clinical trial conducted by Bollo et al., the colon length was also longer in the IA group than in the EA group (25.3 vs. $22.7 \mathrm{~cm}, p=0.026$ ) [10]. In the subgroup analysis, we noted no significant difference in colon length when the lesions were in the cecum or appendix. Interestingly, the further the lesion was from the cecum, the greater the difference in colon length was between the IA and EA groups. When the tumor was located at the hepatic flexure or transverse colon, the colon length and nearest margin were greater when using the IA method. This finding may be explained by the greater visualization of the bowel, achieving a more precise incision and minimizing bowel compromise during resection by avoiding the need to exteriorize a thick specimen through a small laparotomy incision with IA.

To date, there is only one study that compared the postoperative laboratory data of the IA and EA groups. Mari et al. compared preoperative and serial postoperative 
serum inflammatory markers between 30 patients who underwent a TLC and 30 patients who underwent a LAC and found significantly lower interleukin-6, CRP, and WBC levels on postoperative days 1,3 , and 5 in the IA group [21], indicating that the IA group had a lower surgical stress response that might play a role in bowel recovery. In contrast, this study revealed significantly higher WBC and CRP levels on postoperative day 3 in the IA group. Although these high inflammatory markers were present, there was no difference in the incidence of postoperative complications, such as intra-abdominal infection, abscess formation, wound infection, or anastomosis insufficiency, between the groups. The incidence of postoperative ileus was also comparable between the two groups, suggesting that the risk of infection and postoperative complications was not increased when opening the intestinal tract during IA.

In this study, the mean LOS after surgery was significantly shorter in the IA group, especially a LOS less than 5 days. The shorter LOS represents an overall better recovery after surgery with the IA method, which includes less wound pain, less stress on the bowel, and better recovery of bowel function. Many systemic reviews and meta-analyses have demonstrated similar findings [22-27]. The IA method has the following advantages: lower analgesic use, faster time to bowel movement and flatus passage, shorter time to solid food intake, and shorter length of hospitalization. The postoperative complications were comparable between the two methods [7, 8, 10, 24-27].

Hanna et al. demonstrated comparable 5-year overall survival ( $66 \%$ vs. $78 \%, p=0.698)$ and disease-free survival ( $86 \%$ vs. $78 \%, p=0.999)$ between the IA and EA groups, comprising of 195 patients undergoing a laparoscopic right hemicolectomy [12]. Another study by Lee et al. also showed comparable overall survival $(71 \%$ vs. $76 \%, p>0.05)$ and disease-free survival ( $82 \%$ vs. $85 \%, p$ $>0.05$ ) between the IA and EA groups at the 3-year follow-up [7]. In the present study, no significant difference in overall survival and disease-free survival was observed between the IA group and EA group. It is of concern that tumor cell dissemination while opening the intestinal tract in an environment of high-pressure pneumoperitoneum may cause an increased rate of peritoneal recurrence. To survey whether peritoneal recurrence is increasing because of opening the intestinal tract during the IA procedure, we focused on the occurrence of peritoneal seeding during the follow-up. Among all participant studies, there were equivalent numbers of peritoneal seeding detectable by CT/PET survey and/or re-operation between the IA and EA groups, indicating no increased risk of tumor cell dissemination even if we open the intestinal tract inside the peritoneal cavity. Ambe et al. analyzed the cytology specimens acquired from the endoscopic retrieval bag after intracorporeal resection of the colorectal cancer laparoscopically and found no malignant cells. Furthermore, peritoneal recurrence was not observed during the 14-month follow-up [28]. We noted that the primary $\mathrm{T}$ stage of the resected tumor in our series was T3 or T4, which may imply that the tumor factor itself is more critical than the surgical factor.

The strength of this study is that, to our knowledge, we are the first to compare, in detail, the length of specimens and margins according to the tumor location between patients who underwent IA and those who underwent EA to confirm the hypothesis that IA offers a precise resection of the specimen. Moreover, we are the first to compare whether peritoneal recurrence is increased with the IA procedure to answer the question whether surgeons should avoid opening the intestinal tract during the surgery for better oncological results. This study is limited by its retrospective nature; however, by applying PSM, the demographic characteristics of both groups used in the analysis were the same. Another limitation in this study is the small sample size and the oncological results may not be as reliable as larger trials. Long-term results by larger prospective trials are desirable to confirm these findings.

\section{Conclusions}

In conclusion, laparoscopic right hemicolectomy with IA offers a variety of wound incision sites for tumor retraction, achieves better postoperative recovery without increasing the incidence of complications, and allows for a more precise incision that achieves a better surgical margin. The overall survival, disease-free survival, and the rate of peritoneal recurrence were comparable between IA and EA procedures. Thus, IA can be considered a safe procedure for patients with right-sided colon lesions.

\section{Abbreviations}

LAC: Laparoscopy-assisted colectomy; TLC: Total laparoscopic colectomy; IA: Intracorporeal anastomosis; EA: Extracorporeal anastomosis;

ASA: American Society of Anesthesiologists; PSM: Propensity score matching; NOSE: Natural orifice specimen retraction; LOS: Length of stay;

CEA: Carcinoembryonic Antigen; PET: Positron emission tomography

\section{Acknowledgements}

None.

Authors' contributions

$\mathrm{CL}$ and $J Y$ conceived the study design. $\mathrm{YL}, \mathrm{YH}$, and $\mathrm{YC}$ acquired the data for the study. WT, PH, and $\mathrm{HH}$ analyzed and interpreted the data. $\mathrm{CL}$ drafted the manuscript. CY and JC revised the manuscript critically. The authors read and approved the final manuscript.

\section{Funding}

This study was not funded by any outside source

Availability of data and materials

The datasets generated and analyzed during the current study available from the corresponding author on reasonable request. 


\section{Ethics approval and consent to participate}

The study protocol was approved by the institutional review board of Taoyuan branch of Chang Gung Memorial Hospital as approval number 202000106BO. Due to the retrospective design of the study, the local ethic committee confirmed that informed consent was not necessary from participants.

\section{Consent for publication}

Not applicable.

\section{Competing interests}

The authors declare that they have no competing interests

Received: 12 October 2020 Accepted: 10 December 2020 Published online: 04 January 2021

\section{References}

1. The COLOR Study Group. COLOR: a randomized clinical trial comparing laparoscopic and open resection for colon cancer. Dig Surg. 2000;17:617-22..

2. Lacy AM, García-Valdecasas JC, Delgado S, et al. Laparoscopy-assisted colectomy versus open colectomy for treatment of non-metastatic colon cancer: a randomised trial. Lancet. 2002;359:2224-9.

3. Clinical Outcomes of Surgical Therapy Study Group, Nelson H, Sargent DJ, et al. A comparison of laparoscopically assisted and open colectomy for colon cancer. N Engl J Med. 2004;350:2050-9.

4. Colon Cancer Laparoscopic or Open Resection Study Group, Buunen M, Veldkamp R, et al. Survival after laparoscopic surgery versus open surgery for colon cancer: long-term outcome of a randomised clinical trial. Lancet Oncol. 2009;10:44-52.

5. Bergamaschi R, Schochet E, Haughn C, et al. Standardized laparoscopic intracorporeal right colectomy for cancer: short-term outcome in 111 unselected patients. Dis Colon Rectum. 2008:51:1350-5.

6. Grams J, Tong W, Greenstein AJ, Salky B. Comparison of intracorporeal versus extracorporeal anastomosis in laparoscopic-assisted hemicolectomy. Surg Endosc. 2004;24:1886-91.

7. Lee $\mathrm{KH}, \mathrm{Ho}$ J, Akmal Y, et al. Short- and long-term outcomes of intracorporeal versus extracorporeal ileocolic anastomosis in laparoscopic right hemicolectomy for colon cancer. Surg Endosc. 2013;27:1986-90.

8. Magistro C, Di Lernia S, Ferrari G, et al. Totally laparoscopic versus laparoscopic-assisted right colectomy for colon cancer: is there any advantage in short-term outcomes? A prospective comparative assessment in our center. Surg Endosc. 2013;27:2613-8.

9. Biondi A, Pietro S, Pennestrì F, et al. Totally laparoscopic right colectomy versus laparoscopically assisted right colectomy: a propensity score analysis. Surg Endosc. 2017;31:1-8.

10. Bollo J, Turrado V, Rabal A, et al. Randomized clinical trial of intracorporeal versusextracorporeal anastomosis in laparoscopic right colectomy (IEA trial). Br J Surg. 2019;107:364-72.

11. Allaix ME, Degiuli M. Bonino, et al.: Intracorporeal or extracorporeal ileocolic anastomosis after laparoscopic right colectomy: a double-blinded randomized controlled trial. Ann Surg. 2019;270(5):762-7.

12. Hanna MH, Hwang GS, Phelan MJ, et al. Laparoscopic right hemicolectomy: short- and long-term outcomes of intracorporeal versus extracorporeal anastomosis. Surg Endosc. 2015;30:1-10.

13. Shapiro R, Keler U, Segev L, et al. Laparoscopic right hemicolectomy with intracorporeal anastomosis: short- and long-term benefits in comparison with extracorporeal anastomosis. Surg Endosc. 2016;30:1-7.

14. Cleary RK, Kassir A, Johnson CS, et al. Intracorporeal versus extracorporeal anastomosis for minimally invasive right colectomy: a multi-center propensity score-matched comparison of outcomes. PLoS One. 2018;13: e0206277.

15. Singh R, Omiccioli A, Hegge S, McKinley C. Does the extraction-site location in laparoscopic colorectal surgery have an impact on incisional hernia rates? Surg Endosc. 2008;22:2596-600.

16. Orcutt ST, Balentine CJ, Marshall CL, et al. Use of a Pfannenstiel incision in minimally invasive colorectal cancer surgery is associated with a lower risk of wound complications. Tech Coloproctol. 2012;16:127-32.

17. Benlice C, Stocchi L, Costedio MM, et al. Impact of the specific extractionsite location on the risk of incisional hernia after laparoscopic colorectal resection. Dis Colon Rectum. 2016;59:743-50.
18. Mimica Z, Pogorelić Z, Perko Z, et al. Effect of surgical incision on pain and respiratory function after abdominal surgery: a randomized clinical trial. Hepatogastroenterology. 2007;54:2216-20.

19. Kisielinski K, Conze J, Murken AH, et al. The Pfannenstiel or so called "bikini cut": still effective more than 100 years after first description. Hernia. 2004;8: $177-81$.

20. Milone M, Elmore U, Di Salvo E, et al. Intracorporeal versus extracorporeal anastomosis. Results from a multicentre comparative study on 512 rightsided colorectal cancers. Surg Endosc. 2015;29:1-7.

21. Mari GM, Crippa J, Costanzi ATM, et al. Intracorporeal anastomosis reduces surgical stress response in laparoscopic right hemicolectomy: a prospective randomized trial. Surg Laparosc Endosc Percutan Tech. 2018;28:77-81.

22. Feroci F, Lenzi E, Garzi A, et al. Intracorporeal versus extracorporeal anastomosis after laparoscopic right hemicolectomy for cancer: a systematic review and meta-analysis. Int J Colorectal Dis. 2013:28:1177-86.

23. van Oostendorp S, Elfrink A, Borstlap W, et al. Intracorporeal versus extracorporeal anastomosis in right hemicolectomy: a systematic review and meta-analysis. Surg Endosc. 2016;31:64-77.

24. Wu Q, Jin C, Hu T, et al. Intracorporeal versus extracorporeal anastomosis in laparoscopic right colectomy: a systematic review and meta-analysis. J Laparoendosc Adv Surg Tech. 2017;27:348-57.

25. Milone M, Elmore U, Vignali A, et al. Recovery after intracorporeal anastomosis in laparoscopic right hemicolectomy: a systematic review and meta-analysis. Langenbecks Arch Surg. 2017:403:1-10.

26. Vergis A, Steigerwald S, Bhojani F, et al. Laparoscopic right hemicolectomy with intracorporeal versus extracorporeal anastomosis: a comparison of short-term outcomes. Can J Surg. 2015;58:63-6.

27. Widmar M, Aggarwal P, Keskin M, et al. Intracorporeal anastomoses in minimally invasive right colectomies are associated with fewer incisional hernias and shorter length of stay. Dis Colon Rectum. 2020;63:685-92.

28. Ambe PC, Kankam J, Zarras K. Peritoneal spillage is not an issue in patients undergoing minimally invasive surgery for colorectal cancer. World I Surg Oncol. 2020:18(1):107.

\section{Publisher's Note}

Springer Nature remains neutral with regard to jurisdictional claims in published maps and institutional affiliations.

Ready to submit your research? Choose BMC and benefit from:

- fast, convenient online submission

- thorough peer review by experienced researchers in your field

- rapid publication on acceptance

- support for research data, including large and complex data types

- gold Open Access which fosters wider collaboration and increased citations

- maximum visibility for your research: over $100 \mathrm{M}$ website views per year

At BMC, research is always in progress.

Learn more biomedcentral.com/submissions 